\title{
RAPID AND SLOW COMPONENTS OF THE CIRCULATION IN THE HUMAN FOREARM ${ }^{1}$
}

\author{
BY EDWARD D. FREIS, HAROLD W. SCHNAPER, AND \\ LAWRENCE S. LILIENFIELD 2 \\ (From the Cardiovascular Research Laboratory, Georgetown University School of Medicine \\ and the Veterans Administration Hospital, Washington, D. C.)
}

(Submitted for publication April 20, 1956; accepted October 1, 1956)

The present study is concerned with the manner in which a segment of arterial blood flows through the vessels of the human forearm. Specifically it is directed toward answering the question whether blood flows as a unit or is distributed into channels with varying rates of flow. The experimental procedure employed has as its basis the fact that the forearm circulation is small in comparison to that of the total body.

Volume dilution methods as commonly employed for measuring either cardiac output or total blood volume require a dose of dye or other labelling material sufficiently large to produce significant concentrations in the general circulation. In the cardiac output method only the upslope, peak and first portion of the downslope may be obtained before distortion occurs due to contamination with recirculating labelled material.

In the forearm, however, only a small quantity of dye or other labelling substances injected into the brachial artery produces easily measurable concentrations in the effluent veins. The portion of this small dose of dye which escapes into the general circulation becomes so well diluted that its concentration in vessels elsewhere in the body cannot be detected by ordinary spectrophotometric methods. Hence, the washout of the dyed segment of forearm arterial blood can be studied without contamination or distortion by significant amounts of recirculating injectate.

The purpose of this investigation was not to measure absolute blood flow of the forearm and hand. The validity of indicator-dilution methods has not been established for making such measurement in a peripheral area which has multiple ve-

\footnotetext{
1 Supported in part by research grants from the $\mathrm{Na}$ tional Heart Institute, National Institutes of Health, U. S. Public Health Service and the Squibb Institute for Medical Research, New Brunswick, N. J.

2 Helen H. Millenson Memorial Fellow, Metropolitan Heart Guild, Washington, D. C.
}

nous drainage (1). Rather, the concern was with the characteristics of the uncontaminated downslope of the labelled blood in the area drained by the particular effluent vein being sampled.

\section{MATERIALS AND METHODS}

The subjects either were normal or patients on the hospital wards. They were all young or early middleaged males. All patients were afebrile and ambulatory for at least one week prior to serving as subjects for these experiments. None were suffering from diseases of the cardiovascular system.

The method was similar to that described in a previous communication (2) except that the amount of dye injected was smaller. One-fourth $\mathrm{ml}$. of a 0.5 per cent solution of the blue dye T-1824 was diluted in a mixture containing $3.5 \mathrm{ml}$. of deuterium oxide in saline and 0.2 $\mathrm{ml}$. of 5 per cent sodium thiocyanate in distilled water. Three $\mathrm{ml}$. of this mixture was injected into the brachial artery through a 20 gauge needle attached to a threeway stopcock. Thiocyanate and deuterium oxide were added to the mixture in order to study the time-concentration curves of these permeable substances, as will be described in future reports.

Immediately following the injection sampling was begun through a 17 gauge "thin-walled" needle threaded well into a large antecubital vein. Whenever possible a vein was chosen which appeared to drain the deep as well as the superficial structures. Samples were collected in the manner described previously (2), usually at intervals of 2 seconds, then 20 -second intervals to 4 minutes, then $5,6,8,10,12$ and 15 minutes, respectively. A sample was withdrawn from a vein in the opposite arm 3 to 5 minutes after the injection in order to rule out the possibility of significant amounts of recirculating labelled material.

Several precautions were necessary to insure valid results. The needle in the vein was directed against the stream of flow and the bevel so placed as to provide good outflow and adequate samples. Scanty samples resulted when the bevel was against the side of the vein, producing distorted curves probably due to delay and mixing in the needle and collecting catheter. In cases in which an adequate outflow could not be obtained (less than $0.5 \mathrm{ml}$. of blood per second) the results were discarded. The arterial needle also must be well placed, permitting unobstructed injection of the tracer materials, 
and the injection must be accomplished evenly, gently and rather slowly $(0.5$ to $1.0 \mathrm{ml}$. per second) in order to avoid arterial spasm. Arterial spasm was recognized by a sudden temporary decrease in outflow from the venous drainage needle. When this occurred the results were discarded.

The dye concentrations in the plasma were determined spectrophotometrically using $1-\mathrm{ml}$. cuvettes. It usually was necessary to dilute the samples containing the peak dye concentrations (and the plasma blank) with saline in order to obtain accurate readings. Occasional hemolyzed samples either were discarded or were read after precipitating the proteins of the sample and plasma blank with acetone. The resulting dye density values were plotted on semi-log paper. Chromium labelled red cells were prepared and the radioactivity of the samples was determined by the method of Sterling and Gray (3).

Mean circulation time was calculated as follows: each concentration per unit time was multiplied by its re- spective time from the midpoint of injection. The sum of these products then was divided by the sum of the concentrations. The volume of the collecting system (needle, stopcock and collecting catheter) was measured and appropriate correction made for delay in the catheter.

\section{RESULTS}

Uncontaminated curves were obtained in 28 subjects, in 25 with the dye T-1824 and in 3 with $\mathrm{Cr}^{51}$ labelled red cells (Table I). A monophasic downslope was obtained in only 3 of these cases. In the remaining 22 patients receiving the dye and in the 3 cases receiving $\mathrm{Cr}^{51}$ labelled red cells the downslopes were biphasic. Representative biphasic curves are illustrated in Figure 1. After injection there was a brief, latent period following

TABLE I

Mean circulation times and ratios of flows and volumes in the forearm circulation

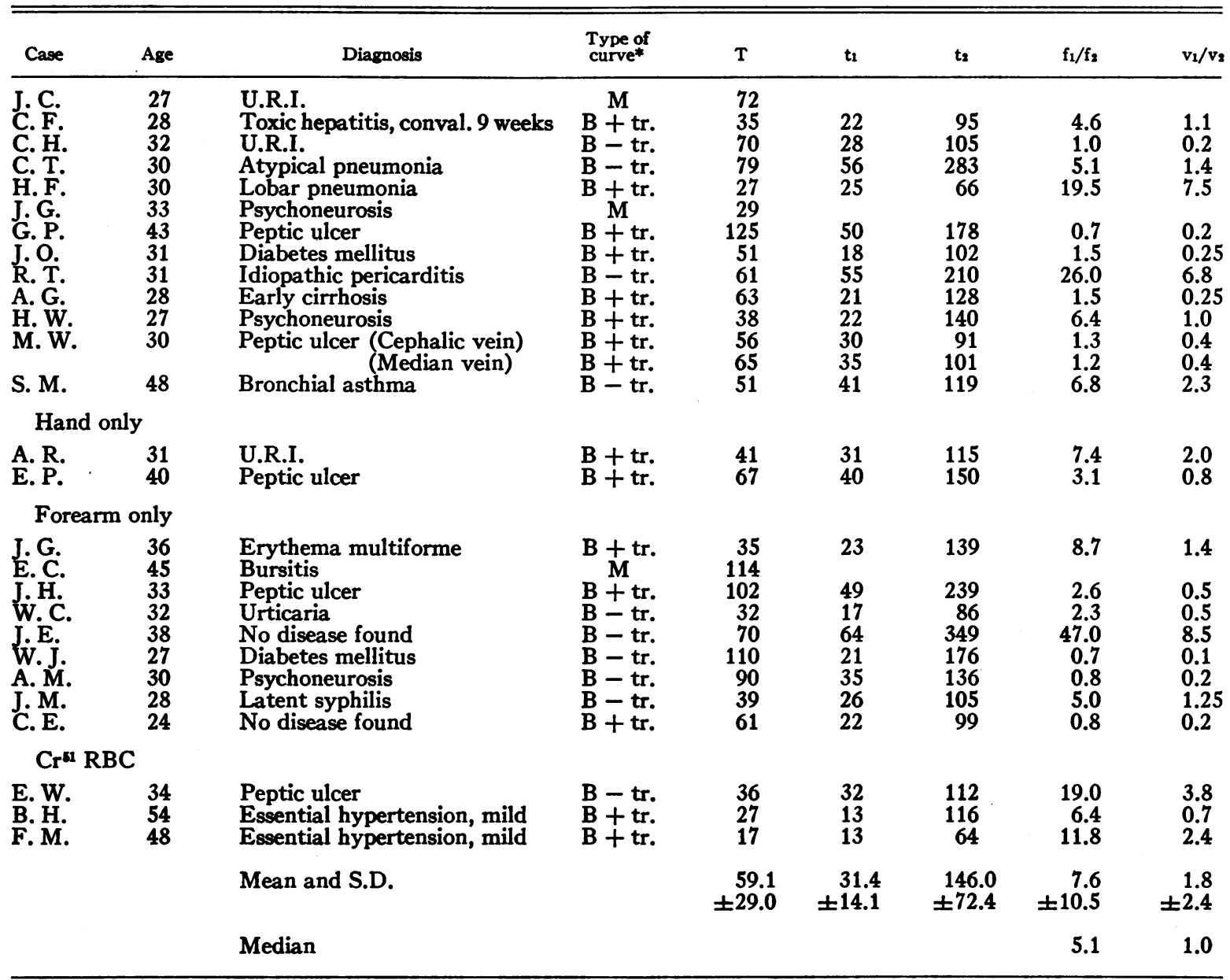

* $\mathrm{M}=$ Monophasic downslope; $\mathrm{B}+\mathrm{tr}$. = Biphasic downslope with transition zone; B - tr. = Biphasic downslope without transition zone. 
which the dye appeared and rapidly increased in concentration forming a steep ascending phase lasting generally a few seconds. The curve fell away at first rapidly and then more slowly. The transition between the early and late phases of the downslope either was abrupt or connected by a short transition zone. The two phases of the downslope both were exponential forming straight lines when plotted on semi-log paper. In 10 cases the early steep downslope and later shallower downslope were connected directly without an intervening transition zone (Figure 1a). In the remaining 15 subjects a transition zone which was intermediate in gradient connected the early and late phases of the downslope (Figure 1b).

Although the majority of the curves showed clearly delineated biphasic downslopes there were some variations from the type of curves illustrated. The variations seen included irregularity of upslope, "sawtooth" or double peaks and small, late, third phase which was either shallower or steeper than the preceding phase. These variations usually were observed in the cases in which small samples were obtained presumably because of poor venous blood flow. In the calculations which follow, these variations were "rounded off" because they would be difficult to subject to mathematical treatment and were not representative of the majority of the curves.

The biphasic downslopes were not due to differences in velocity of blood flow through the hand as compared with the forearm. In 9 subjects the hand was excluded by inflating a cuff on the wrist to pressures $100 \mathrm{~mm}$. $\mathrm{Hg}$ above systolic pressure (Table I). Typical biphasic downslopes were seen in 8 of these cases. In two subjects (A. R. and E. P., Table I) the injection was made into the radial artery and sampling was carried out through a vein in the wrist. Multiphasic downslopes were obtained in both instances.

\section{Analysis of the biphasic downslope into compo- nents with different flows and volumes}

Reasons are presented in the discussion for believing that the biphasic downslopes represent a rapid flow component producing the early steep downslope and a slower component producing the later shallow downslope. It was desirable to analyze these component parts quantitatively in terms
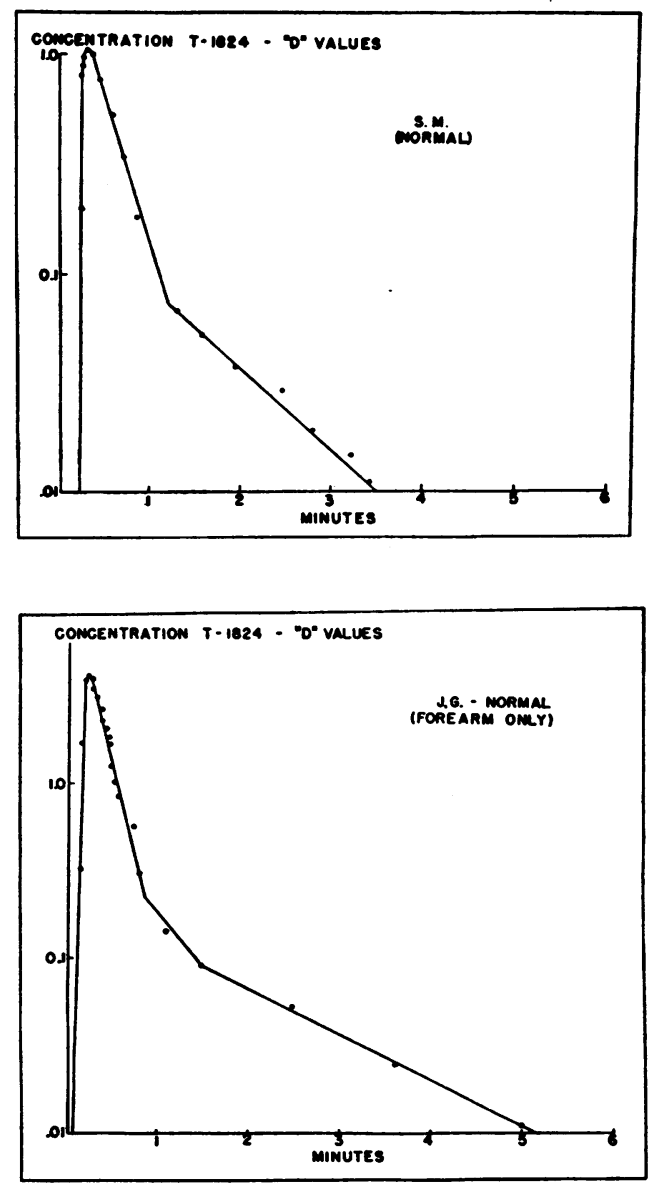

Fig. 1. Graphs Illustrating Typical Biphasic Time-Concentration Washout Slopes in the Human FOREARM

Figure 1a (above) illustrates a simple biphasic exponential curve. Figure $1 \mathrm{~b}$ (below) shows a biphasic curve with transition zone.

of flow and volume. Absolute measurements of flow were not possible because the dye is not always distributed uniformly in the forearm (1). However, the relative flows and relative volumes in the biphasic system could be determined using only the mean circulation times.

The solution was based on the well known relationship that vascular volume from the point of injection to the point of sampling is equal to blood flow multiplied by the mean circulation time. Thus, $v_{1}$, the vascular volume of the rapid component, was equal to $f_{1}$ (the flow in this component) times $t_{1}$ (its mean circulation). Similarly, $v_{2}$ (the volume of the slow component) equalled $f_{2}$ times $t_{2}$, and $V$ (the total vascular volume of the seg- 


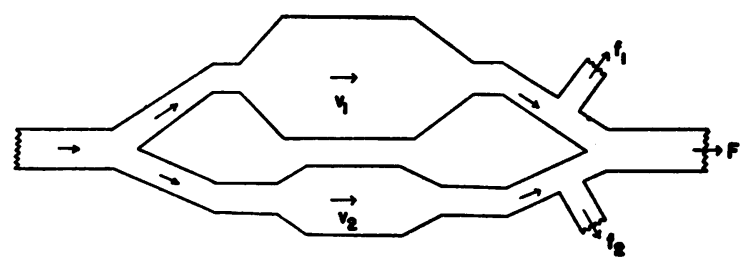

Fig. 2. Diagram of Model Built from Plastic Cylinders and Tygon Plastic Tubing

See text for details.

ment of forearm drained by the vein being sampled) equalled $F$ times $T$ (the mean circulation time of the entire curve).

Thus:

$$
\begin{aligned}
& v_{1}=f_{1} t_{1}, \\
& v_{2}=f_{2} t_{2}, \\
& V=F T .
\end{aligned}
$$

It also was assumed that:

$$
\begin{aligned}
\mathrm{v}_{1}+\mathrm{v}_{2} & =\mathrm{V}, \\
\mathrm{f}_{1}+\mathrm{f}_{2} & =\mathrm{F} .
\end{aligned}
$$

The ratio $f_{1} / f_{2}$ was obtained as follows:

$$
\begin{aligned}
f_{1}=\frac{v_{1}}{t_{1}}= & \frac{V-v_{2}}{t_{1}}=\frac{F T-f_{2} t_{2}}{t_{1}} \\
= & \frac{F T-\left(F-f_{1}\right) t_{2}}{t_{1}}=\frac{F T-F t_{2}+f_{1} t_{2}}{t_{1}}, \\
& f_{1} t_{1}-f_{1} t_{2}=F T-F t_{2}
\end{aligned}
$$

or

$$
\begin{gathered}
f_{1}\left(t_{1}-t_{2}\right)=F\left(T-t_{2}\right), \\
f_{1}=\frac{F\left(T-t_{2}\right)}{t_{1}-t_{2}}
\end{gathered}
$$

Similarly:

$$
\begin{gathered}
f_{2}=\frac{F\left(T-t_{1}\right)}{t_{2}-t_{1}}, \\
\frac{f_{1}}{f_{2}}=\frac{t_{2}-T}{T-t_{1}}, \text { and } \frac{v_{1}}{v_{2}}=\left(\frac{f_{1}}{f_{2}}\right)\left(\frac{t_{1}}{t_{2}}\right) .
\end{gathered}
$$

A simple model was prepared to test these assumptions (Figure 2). Plastic cylinders with gently sloping inflow and outflow tracts designed to minimize areas of stagnant flow and permit good mixing of the dye were designated $v_{1}$ and $\mathbf{v}_{2}$. These were filled loosely with glass wool to further promote adequate mixing. These plastic vessels were connected with Tygon plastic tubing as shown in Figure 2 and the inlet was connected to a constant overflow reservoir. Tap water was per- mitted to flow through the system and T-1824 was injected into the common inflow tract.

By placing a resistance (screw clamp) in the inflow tract to either $v_{1}$ or $v_{2}$ the flows to either $v_{1}$ or $v_{2}$ could be adjusted at will. By clamping the common outflow tubing the flows through $v_{1}$ and $v_{2}$ could be measured separately either directly or by the dye dilution method. After several such determinations the side channels were clamped and the common outflow tubing opened. The dye again was injected in order to obtain the composite dye dilution curve.

One such experiment is illustrated in Figure 3. The solid lines represent the curves obtained from $v_{1}$ and $v_{2}$ separately. The broken line is the curve obtained when the two volume flows were permitted to mix and flow out of the common channel. After extrapolating the downslope of the first portion of the broken line curve and the upslope of the second portion $t_{1}, t_{2}$ and $T$, the respective mean circulation times, were measured, and from these the ratios of the flows and volumes. The ratio of flows indicated that $f_{1}$ was 6 times $f_{2}$ in comparison to the actual ratio of 8.5 times. The ratio of the volumes indicated that $v_{1}$ was twice $v_{2}$,

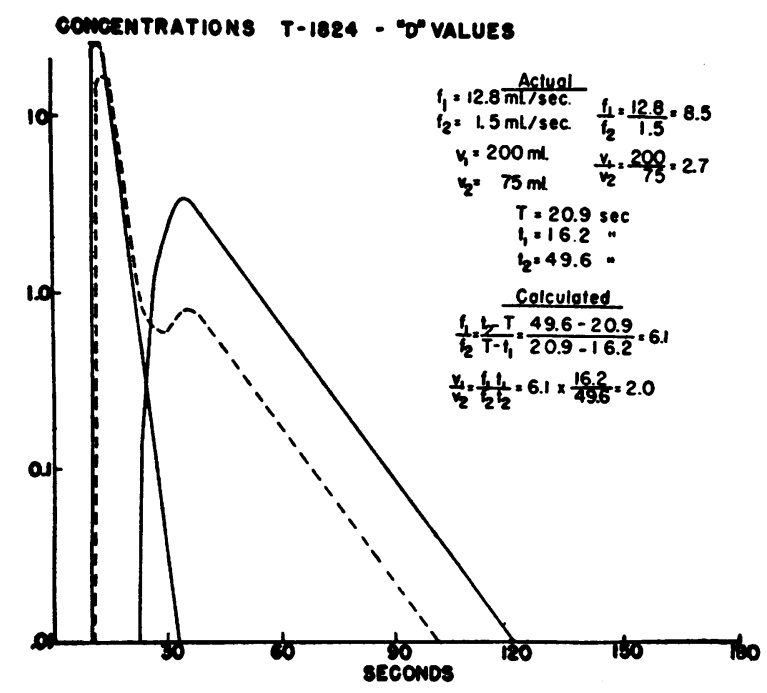

Fig. 3. Chart of T-1824 Time-Concentration Curves Obtained by Permitting Tap Water to Flow Through the Model System Illustrated IN Figure 2

The solid line curves were obtained by collecting the outflow from $v_{1}$ and $v_{2}$ separately. The broken line represents the curve obtained by collecting through the common outflow tubing. See text for details. 


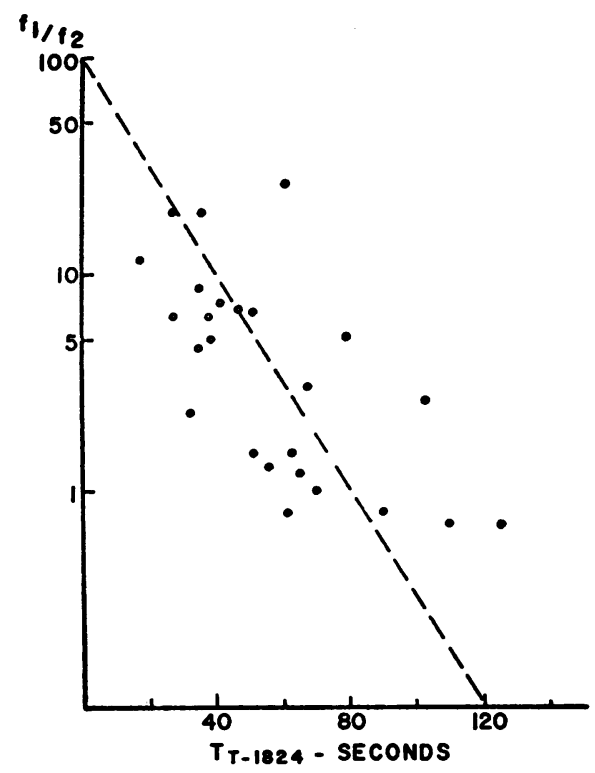

Fig. 4. Graph Showing the Ratio $f_{2} / f_{2}$ Plotted on Log Scale Against linear Plot of the Mean Circulation Time (T) of the Enttre Curve

$f_{1} / f_{2}$ increases inversely to $T$.

which was a reasonable approximation of the actual value of 2.7 times.

It seemed probable that a closer approximation to actual values could have been obtained by taking greater pains to ensure adequate mixing and by making the system larger in order to prolong the collection period. However, we were satisfied that extrapolation of the composite biphasic curve gave a reasonable approximation of the two monophasic curves which represented the volume and flow in the constituent compartments.

\section{Ratios of flows and volumes in the biphasic system of the forearm}

The curves of the biphasic downslope were extrapolated by first projecting downward the straight line early phase of the downslope and then subtracting this extrapolated portion from the corresponding values of the second portion (or transition zone if present) of the actual downslope. The values obtained by such subtraction were plotted as the upslope of the second portion.

$T$, the mean circulation time of the entire curve, ranged between seventeen and 125 seconds (mean $59 \pm 29$ seconds) in the 28 subjects (Table I).
In the 25 cases exhibiting biphasic downslopes the mean circulation time of the first or rapid component $\left(t_{1}\right)$ averaged $31.4 \pm 14.1$ seconds with a range from 13 to 64 seconds. The mean circulation time of the second or slower component $\left(t_{2}\right)$ averaged $146.0 \pm 72.4$ seconds and ranged from 64 to 349 seconds.

Flow was greater in the rapid component in all cases except 4 , the mean of $f_{1} / f_{2}$ being $7.6 \pm 10.5$. As indicated by the large standard deviation the range was great, the values varying from 0.7 to 47 . The volume of the rapid component was greater than that of the slow portion in only 11 of the 25 cases. The mean of $\mathrm{v}_{1} / \mathrm{v}_{2}$ was $1.8 \pm 2.4$ with a range of 0.1 to 8.5. Because of distortion of the means by a few extremely high ratios the medians also were calculated. The median $f_{1} / f_{2}$ was 5.1 and median $\mathrm{v}_{1} / \mathrm{v}_{2}$ was 1.0 .

An approximate inverse relationship existed between $f_{1} / f_{2}$ and $T$. The relationship assumed a straight line function when $f_{1} / f_{2}$ was plotted on a log scale (Figure 4). This relationship implies that in general the greater the blood flow the more $f_{1}$ will predominate over $f_{2}$.

A relationship also was found between $v_{1} / v_{2}$ and $f_{1} / f_{2}$ (Figure 5), $f_{1} / f_{2}$ averaging 6 times $\mathrm{v}_{1} / \mathrm{v}_{2}$ with a range of 2.5 to 10.5 times including all but two values. This indicates that the volumes of the two components vary in such a way that as flow in the rapid component increases so does its relative volume.

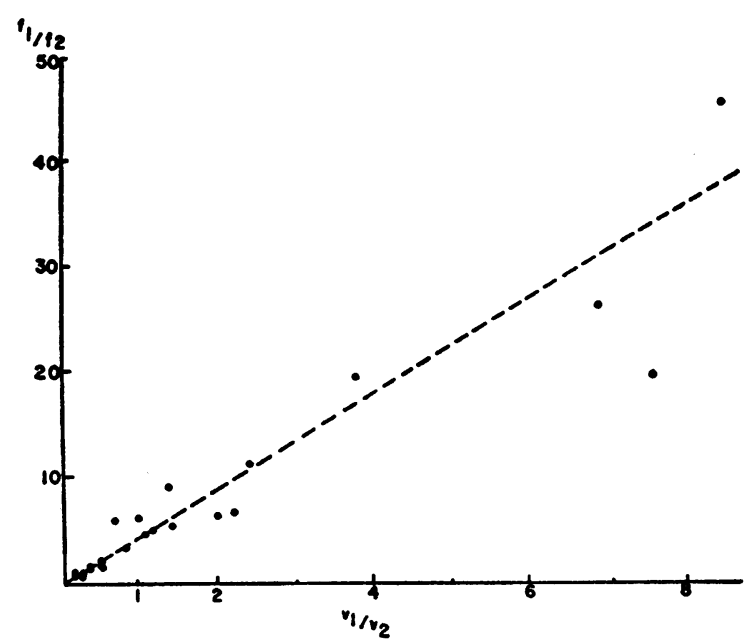

Fig. 5. Graph Showing the Relationship Between $\mathrm{f}_{1} / \mathrm{f}_{\mathbf{2}}$ AND $\mathbf{v}_{1} / \mathrm{v}_{\mathbf{2}}$ 


\section{DISCUSSION}

Several hypotheses can be advanced to explain the observed biphasic system. The first to be considered is differences in path lengths. According to Wiggers (4) the velocity of blood flow in the various subdivisions of the vascular tree varies inversely with the cross sectional area, decreasing gradually in the arteries from an aortic velocity of $18 \mathrm{~cm}$. per second, slowing abruptly in the arterioles and capillaries where velocities of approximately $5 \mathrm{~mm}$. per second exist, and increasing again in the large veins to 5 or more $\mathrm{cm}$. per second. Assuming the longest probable path length in the forearm to be $80 \mathrm{~cm}$. for both arterial and larger venous segments, $4 \mathrm{~cm}$. for the arteriole, $2 \mathrm{~cm}$. for the capillary, $4 \mathrm{~cm}$. for venule and 10 $\mathrm{cm}$. for the small venous segments, and employing conservative estimates of velocity in these segments, transit through the longest pathway would be less than 30 seconds. This fails to agree with the mean circulation times observed in the present experiments, since mean $t_{2}$ was 141 seconds or approximately 5 times the duration required for blood to flow through the longest pathway on the basis of presently assumed mean velocities (4).

The actual distribution of path lengths in the forearm would be expected to follow a typical population or "bell-shaped" distribution curve. If the velocities of flow were uniform in each subdivision throughout the forearm and difference in path lengths was the only variable, the downslope would be monophasic, the ascending limb representing the shortest pathways, the peak the numerically greatest path lengths and the downslope the longest pathways. In order to explain the presently observed biphasic system two distinct populations of pathways are assumed, both being present in the hand alone and in the forearm alone.

If path length was the only variable, and since $t_{2}$ averaged more than 4 times $t_{1}$, it also is assumed that the 2 populations of path lengths differed from each other by a factor of 4 . On the basis of presently accepted circulatory velocities (4) the capillaries composing the slow compartment would average about $100 \mathrm{~cm}$. in length, a figure which seems to be incredibly large.

The two compartment system also might be ex- plained on the basis of differences in blood velocity through the principal tissues of the forearm, namely, muscle and skin. Blood velocity through the skin alone can be estimated by observing directly the passage of appropriate doses of fluorescein or T-1824 in the forearm following brachial arterial injection (5). Peak dye concentrations occur within 10 to 30 seconds after the dye is injected, although traces can be seen in heavily stained areas for longer periods. These studies indicate that the major portion of blood flow to the skin travels at velocities consistent with $t_{1}$ and not $t_{2}$. Thus, if the slow component is limited to one of the major tissue subdivisions of the forearm, it probably resides in the muscles.

Data to be presented later do not support this explanation. Local muscular exercise to the point of exhaustion was carried out by having the subject repeatedly squeeze a hand dynamometer. Dye curves following such local exercise revealed proportionate reductions of both $t_{1}$ and $t_{2}$ compared with the controls. Since muscle and not skin was affected predominantly by such exercise, and if muscle were the site of the slow component, there should have been a disproportionate reduction of $t_{2}$. In addition, in the untreated subject biphasic curves were observed in the hand alone, which contains little muscle, and in forearm alone (exclusive of the hand and wrist), which contains predominantly muscular tissue.

Renkin, utilizing a different technique, observed similar biphasic blood flow patterns in the perfused hindleg of the cat (6). The relative volumes of the two components were uninfluenced by removing the skin. The proportion of bone was too small to account by itself for one compartment and Renkin concluded that both compartments are present in skeletal muscle. These various observations make it appear unlikely that the twophase velocity system is produced by differences in blood velocity in different tissues.

Barcroft presents evidence pointing toward a double circulation in the human forearm and calf (7). He believes that one is under neurogenic control, the other under the influence of local metabolites. The measurements were determined on mean blood flow and its response to various stimuli.

Whereas in the normal subjects flow in the rapid component usually predominated, the vas- 

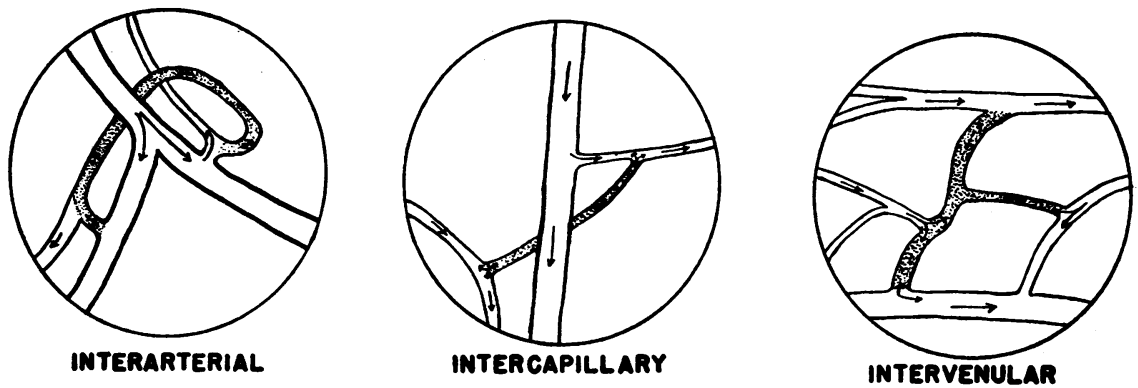

Fig. 6. Typical Anastomotic Connections Between Arterioles, Capillaries and Venules as Observed in the Hamster Cheer Pouch Preparation

Arrows indicate the direction of flow. Arrows with heads pointing in opposite directions and stipled sections indicate anastomotic vessels with sluggish and reversible flow.

cular volumes of the two components were of the same magnitude. Thus, nearly half and in many of the subjects more than half of the vascular volume of the forearm contained slowly circulating blood. As has been outlined above the evidence pointed away from localizing rapid blood flow to one type of tissue and sluggish flow to another and suggested that the two flow systems existed side by side in both skin and muscle.

In seeking for the location of this large vascular component we have observed the circulation in the hamster cheek pouch according to the method of Lutz and Fulton (8). Using 100 to 300 times magnifications it was apparent immediately that the small vessel circulation has a biphasic pattern of blood velocity. In every field vessels of the same order were seen with widely varying rates of blood flow (Figure 6).

The mechanism for the variation of velocity was apparent. The circulation was highly anastomotic with many interarteriolar, intercapillary and intervenular connections. A similar anastomotic circulation has been described in the bat's wing $(9,10)$, in the subcutaneous tissue of mice $(11)$, in voluntary muscle (12) and in intestinal wall (13). Since pressures at either end of the anastomosis were similar, flow was sluggish in the anastomosis. Indeed, it sometimes would stagnate, surge first one way and then the other and occasionally reverse.

Flow through this network of small vessels also was highly dynamic. A capillary or venule appearing to contain the rapid or main line flow would suddenly change into a sluggish channel. Similarly, slow moving flow in an anastomotic vessel would for short periods become rapid, moving sometimes in one direction, at other times in the opposite direction. Thus, not only do the rapid and slow moving circulations exist side by side but some of the small vessels change their role from time to time, now carrying rapid and at another time sluggish flow. In a system composed of anastomotic channels relatively minor shifts in pressure could easily change the pattern of blood flow. The factors controlling such pressure fluctuations are not completely understood although contraction of arterioles and precapillary sphincters probably play a part. Although these direct observations on the microcirculation of other preparations do not prove that the slow component of the forearm circulation in man is due entirely to retarded flow in anastomotic vessels, they provide a reasonable hypothesis for further study.

An alternative method of calculating the relative flows and volumes would be to treat the two curves as if they were occurring synchronously rather than successively. In this situation the late downslope is extrapolated back to the peak of the primary curve (since the two curves are assumed to be synchronous) and the values so obtained subtracted from the early downslope. This will produce a decrease in the ratios $f_{1} / f_{2}$ and $v_{1} / v_{2}$, i.e., $f_{2}$ and $v_{2}$ will become larger relative to $f_{1}$ and $v_{1}$ (Table II). This method of analysis seems to lack validity for the following reasons: in the present experiments the observations began at the instant of first injection of the tracer material which was confined to the intravascular space. The tracer entered as a bolus and was preceded and followed by undyed blood. In essence, it 
TABLE II

Comparison of $t_{1} / t_{2}, f_{1} / f_{2}$ and $v_{1} / v_{2}$ calculated by doronroard extrapolation of the early downslope (first method) and by backward extrapolation of the late downslope (second method) in eight cases selected at random

\begin{tabular}{|c|c|c|c|c|c|c|c|c|c|}
\hline \multirow[b]{2}{*}{ Case } & \multirow[b]{2}{*}{$\mathbf{T}$} & \multicolumn{4}{|c|}{ First method } & \multicolumn{4}{|c|}{ Second method } \\
\hline & & $t_{1}$ & $t_{2}$ & $f_{1} / f_{2}$ & $\mathbf{v}_{1} / \mathbf{v}_{\mathbf{2}}$ & $t_{1}$ & ts & $f_{1} / f_{2}$ & $\mathbf{v}_{2} / \mathbf{v}_{\mathbf{2}}$ \\
\hline $\begin{array}{l}\text { J. H. } \\
\text { A. G. } \\
\text { W. C. } \\
\text { C. F. } \\
\text { C. H. } \\
\text { A. R. } \\
\text { S. M. } \\
\text { E. P. }\end{array}$ & $\begin{array}{r}102 \\
63 \\
32 \\
35 \\
70 \\
41 \\
51 \\
67\end{array}$ & $\begin{array}{l}49 \\
21 \\
17 \\
22 \\
28 \\
31 \\
41 \\
40\end{array}$ & $\begin{array}{r}247 \\
136 \\
86 \\
102 \\
105 \\
140 \\
119 \\
156\end{array}$ & $\begin{array}{r}2.7 \\
1.7 \\
2.3 \\
5.2 \\
1.0 \\
10.0 \\
6.8 \\
3.2\end{array}$ & $\begin{array}{l}0.5 \\
0.25 \\
0.5 \\
1.1 \\
0.2 \\
2.0 \\
2.3 \\
0.8\end{array}$ & $\begin{array}{l}38 \\
17 \\
14 \\
19 \\
23 \\
30 \\
38 \\
32\end{array}$ & $\begin{array}{r}143 \\
84 \\
52 \\
57 \\
77 \\
96 \\
80 \\
107\end{array}$ & $\begin{array}{l}0.6 \\
0.4 \\
1.0 \\
1.4 \\
0.2 \\
5.0 \\
2.3 \\
1.1\end{array}$ & $\begin{array}{l}0.2 \\
0.1 \\
0.3 \\
0.5 \\
0.1 \\
1.6 \\
1.1 \\
0.3\end{array}$ \\
\hline Mean & 58 & 31 & 136 & 4.1 & 1.0 & 26 & 87 & 1.5 & 0.5 \\
\hline
\end{tabular}

was a transient suddenly introduced into a flowing stream which advanced as a front.

If there are two radically different systems of dye transit time in the forearm it is obvious that the bolus will traverse the rapid component with greater speed than the slow component. As a result the appearance and peak times of the rapid component will precede those of the slow. Mixing will not be simultaneous in the two systems. Therefore, the assumption of synchronous rather than successive peaks does not seem to be applicable to this experimental situation.

At first glance it may appear that, since the two components probably are confined to the terminal arborizing network of small vessels, mixing will be simultaneous in both systems. This analysis is not justified, however. The traversal time of the dye is dependent upon the two factors of path length and velocity of flow. Reasons for believing that the biphasic pattern is due to differences in velocity rather than in path length have already been presented. Obviously the same small vessel cannot carry simultaneously both high and low velocity flow. Since the vessels must be separate at any given point in time, the analysis will not be affected whether they present an anastomotic network or two separate mixing chambers as in the model. Each chamber of the model also has to a lesser extent a population of path lengths (the central stream as compared to paths adjacent to the walls of the chamber) and of velocities (laminar flow).

Finally, it should be mentioned that in a few of the biphasic curves a second upslope and peak were evidenced. On the other hand, it must be admitted that the majority of the curves did not show second peaks and that the method of their reconstruction is arbitrary even though based on reasonable assumptions.

It seems probable that a transition zone was present in all of the cases but was not detected in some because it was too short to be apparent without more frequent sampling. The distinction between the presence or absence of a transition zone often was one of personal choice. For example, in Figure 1 it would have been possible to draw a transition zone in patient S. M.

When transition zones were delineated clearly it was found that extrapolation of the early downslope reached an insignificant concentration at a point on the abscissa which approximated the end of the transition zone or beginning of the late downslope. This would be expected if the transition zone represented the merging of the downslope of $t_{1}$ and the upslope of $t_{2}$. However, when the method of analysis dependent upon backward extrapolation of $t_{2}$ was used the resulting $t_{1}$ downslope reached insignificant concentrations at a point in time far short of the end of the transition zone. This would indicate that the contribution of dye from the rapid component had been dissipated at this point. This leaves no explanation for the transition zone except to postulate a relatively brief third component with no transition zones, which seems unlikely. Thus, backward extrapolation of the late downslope leads to serious difficulties in regard to the interpretation of the transition zone and provides an additional reason for discarding this method of analysis in the present experiments.

Regardless of the method of establishing the partition between "fast" and "slow" components the relationship between $f_{1} / f_{2}$ and $T$ as well as $v_{1} / v_{2}$ and $f_{1} / f_{2}$ will be similar qualitatively as can be shown by plotting the representative cases listed in Table II. It should be emphasized that the terms "components" or "compartments" do not imply anatomically discrete systems. As will be shown in a later communication the ratios $f_{1} / f_{2}$ and $v_{1} / v_{2}$ change markedly in the same forearm under conditions of vasodilation, vasoconstriction or venous congestion. These changes in $f_{1} / f_{2}$ and $v_{1} / v_{2}$ continue to be related to $T$ as in the present study. Similar to the hamster cheek 
pouch circulation, therefore, some vessels may at one point in time be members of one component and at another point in time (and especially during major hemodynamic changes) become a part of the other component.

The present biphasic downslopes are not to be confused with the hyperbolic downslopes characteristic of dye curves obtained in a single tube containing laminar flow. The present curves were distinctly biphasic rather than hyperbolic. The "break" in the biphasic curve usually occurred after 60 seconds, which would be quite late if it were due only to the effects of laminar flow. Finally, labelled red cells which are not subjected to laminar flow also exhibited biphasic downslopes.

The question arises whether the biphasic pattern of blood flow is limited to a few body areas or is characteristic of the flow pattern in all organs. Data to be presented in a later communication indicate that the lungs and kidney of the dog exhibit biphasic downslopes although in these rapid flow areas the fast component predominates much more than in the forearm circulation.

\section{SUMMARY AND CONCLUSIONS}

Utilizing a method to prevent significant recirculation, the late downslopes of T-1824 or $\mathrm{Cr}^{51}$ labelled red cells were determined in the human forearm of 28 subjects. . All but three of these curves exhibited biphasic, exponential downslopes.

Analysis of these slopes indicated that the forearm circulation can be divided into a rapid and a slow flowing component. In the normal subject the rapid component usually predominates, whereas the vascular volumes of the two compartments are nearly equal. With increasing forearm blood flow (as indicated by the mean circulation time) flow in the rapid compartment increases relative to that in the slower system. As the relative flow of the rapid component increases its relative volume becomes greater.

Evidence is presented to suggest that the site of the biphasic flow pattern is in the small vessel circulation. Sluggish flow is present in a high percentage of the many interarteriolar, intercapil- lary and intervenular anastomoses that characterize these vessels.

\section{REFERENCES}

1. Andres, R., Zierler, K. L., Anderson, H. M., Stainsby, W. N., Cader, G., Ghrayyib, A. S., and Lilienthal, J. L., Jr., Measurement of blood flow and volume in the forearm of man; with notes on the theory of indicator-dilution and on production of turbulence, hemolysis and vasodilatation by intra-vascular injection. J. Clin. Invest., 1954, 33, 482.

2. Freis, E. D., Stanton, J. R., and Emerson, C. P., Estimation of relative velocities of plasma and red cells in the circulation of man. Am. J. Physiol., 1949, 157, 153.

3. Sterling, K, and Gray, S. J., Determination of the circulating red cell volume in man by radioactive chromium. J. Clin. Invest., 1950, 29, 1614.

4. Wiggers, C. J., Physiology in Health and Disease. 5th ed., Philadelphia, Lea \& Febiger, 1949, p. 595.

5. Partenope, E. A., Freis, E. D., and Lilienfield, L. S., Intermittency of flow in larger vessels of the human forearm. Clin. Research Proc., 1954, 2, 38.

6. Renkin, E. M., Effects of blood flow on diffusion kinetics in isolated, perfused hindlegs of cats. A double circulation hypothesis. Am. J. Physiol., 1955, 183, 125.

7. Barcroft, H., Some problems of innervation in Ciba Foundation. Visceral Circulation. Wolstenholme, G. E. W., Ed., Ciba Foundation Symposium, Boston, Little, Brown \& Co., 1952, p. 167.

8. Lutz, B. R., and Fulton, G. P., The use of the hamster cheek pouch for the study of vascular changes at the microscopic level. Anat. Rec., 1954, 120, 293.

9. Webb, R. L., and Nicoll, P. A., The bat wing as a subject for studies in homeostasis of capillary beds. Anat. Rec., 1954, 120, 253.

10. Nicoll, P. A., and Webb, R. L., Vascular patterns and active vasomotion as determiners of flow through minute vessels. Angiology, 1955, 6, 291.

11. Algire, G. H., and Merwin, R. M., Vascular patterns in tissues and grafts within transparent chambers in mice. Angiology, 1955, 6, 311.

12. Zweifach, B. W., and Metz, D. B., Selective distribution of blood through the terminal vascular bed of mesenteric structures and skeletal muscle. Angiology, 1955, 6, 282.

13. Barlow, T. E., Vascular patterns in the alimentary canal in Ciba Foundation. Visceral Circulation. G. E. W. Wolstenholme, Ed., Ciba Foundation Symposium, Boston, Little, Brown \& Co., 1952, p. 21. 